\title{
Multiple Noises Removal from Computed Tomography (CT) Images
}

\author{
Abdelkader Salama Alrabaie ${ }^{1 *}$, Marwan M M El marmuri², Emhimed Saffor ${ }^{3}$ \\ ${ }^{1}$ Department of Physics, College of Education /Brack, Sebha University, Libya \\ ${ }^{2}$ Department of X-ray Diagnostic \& Radiotherapy, College of Medical Technology, Zawia \\ University, Libya \\ ${ }^{3}$ Department of Physics, College of Sciences, Sebha University, Libya \\ DOI: https://doi.org/10.21467/proceedings.2.3 \\ * Corresponding author email: kadersalama1@gmail.com
}

\begin{abstract}
Noise is seen in images during image acquisition and transmission. This is characterized by noise model. Image enhancement through noise reduction is a fundamental problem in image processing. Noise filtering is a necessary action and has become one of the indispensable components of image processing operation. In this work, we have applied different filtering techniques (arithmetic mean filtering, median, and Weiner) to remove multiple mixed noises such as; (speckle, Gaussian, salt and pepper) from Computed Tomography (CT) images. Performance metrics such as; Peak Signal to Noise Ratio (PSNR), and Mean Square Error (MSE) were used to evaluate filtering techniques. The results showed that the median filter had worked more effectively to remove these noises. All the above mentioned techniques were implemented using MATLAB environment. It should give some results showing the values of PSNR and MSE for these flirters to indicate the differences between them.
\end{abstract}

Keywords: CT, MATL AB, MSE, Noise, PSNR.

\section{Introduction}

Medical images such as magnetic resonance imaging (MRI), computed tomography (CT), ultrasound (US), and X-ray images are subject to a wide variety of distortions, during acquisition, processing, compression, storage, transmission and reproduction, any of which may result in a degradation of visual quality. Medical images are collected by different types of sensors and they are contaminated by different types of noises. Generally speckle; Gaussian, salt and pepper noises mostly occurred in the MRI, CT, and US images.[1] Noise tells unwanted information in digital images. Noise produces undesirable effects such as artifacts, unrealistic edges, unseen lines, corners, blurred objects and disturbs background scenes. In medical image processing many methods are used for noise reduction.[2] Noise filters generally attempt to smooth the corrupted image by neighbourhood operations. To measure the performance and image quality of the noise removal techniques several parameters are available for the comparison. Common parameters are Peak Signal Noise Ratio (PSNR), Root Mean Squared Error (RMSE), Mean Squared Error (MSE) etc.[1][3]

(C) 2018 Copyright held by the author(s). Published by AIJR Publisher in Proceedings of First Conference for Engineering Sciences and Technology (CEST-2018), September 25-27, 2018, vol. 1.

This is an open access article under Creative Commons Attribution-NonCommercial 4.0 International (CC BY-NC 4.0) license, which permits any non-commercial use, distribution, adaptation, and reproduction in any medium, as long as the original work is properly cited. ISBN: 978-81-936820-5-0 
Multiple Noises Removal from Computed Tomography (CT) Images

\section{A). Noise Models:}

i) Gaussian Noise: Gaussian noise caused by natural sources such as thermal vibration of atoms and discrete nature of radiation of warm objects. Gaussian noise generally disturbs the gray values in digital images. That is why Gaussian noise model essentially designed and a characteristic by its probability density function (PDF) or normalizes histogram with respect to gray value and it is given as:[2][4]

$$
P(g)=\sqrt{\frac{1}{2 \pi \sigma^{2}}} \mathrm{e}^{-\frac{(\mathrm{g}-\mu)^{2}}{2 \sigma^{2}}}
$$

Where: $g=$ gray value, $\sigma=$ standard deviation and $\mu=$ mean

ii). Speckle Noise: This noise is multiplicative noise. Their appearance is seen in a coherent imaging system such as laser, radar and acoustics etc. Speckle noise can exist similar in an image as Gaussian noise. Its probability density function follows gamma distribution, and given as:[2]

$$
F(g)=\frac{g^{\alpha-1} e^{\frac{-g}{a}}}{(\alpha-1) ! a^{\alpha}}
$$

Where $\mathrm{a}^{2}$ is the variance, $\alpha$ is the shape parameter of gamma distribution and $g$ is the gray level.

iii). Impulse Valued Noise (Salt and Pepper Noise): Salt and pepper noise is represents itself as randomly occurring white and black pixels. Salt and pepper noise creeps into images in situations where a quick transient, such as faulty switching, takes place.[5] Image pixel values are replaced by corrupted pixel values either maximum or minimum pixel value i.e., 255 or 0 respectively. Although in noisy image, there are possibilities of some neighbours do not

\begin{tabular}{|c|c|c|c|c|c|}
\hline 254 & 207 & 210 & 254 & 207 & 210 \\
\hline 97 & 212 & $\frac{21}{2 t}$ & 197 & 0 & 32 \\
\hline 62 & 106 & 20 & 62 & 106 & 20 \\
\hline
\end{tabular}
changed as shown in Figure 1 for an example.

Figure 1: the central pixel value is corrupted by Pepper noise

$$
p(g)=\left\{\begin{array}{cc}
p a & \text { for } \mathrm{g}=\mathrm{a} \\
p b & \text { for } \mathrm{g}=\mathrm{b} \\
0 & \text { otherwise }
\end{array}\right.
$$

Salt and Pepper noise generally corrupted the digital image by malfunctioning of pixel elements in camera sensors, faulty memory space in storage, errors in digitization process and many more.[2] 
The (PDF) of this noise is shown in the Figure 2.

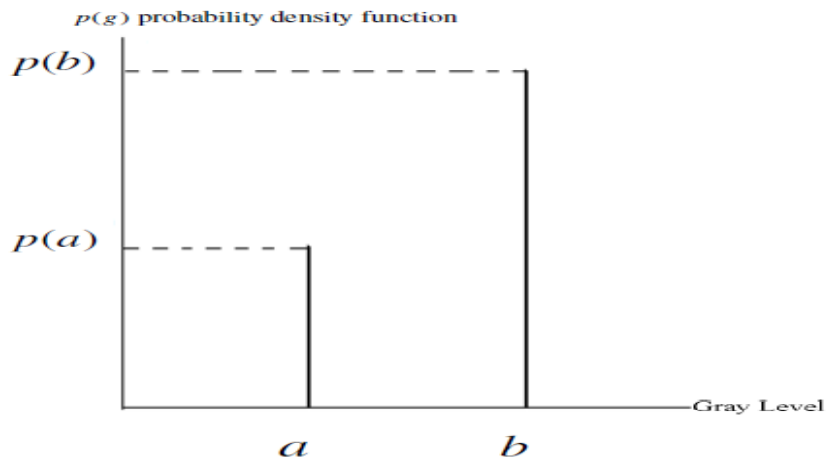

Figure 2: PDF of Salt and Pepper noise

\section{(B) Filters:}

i). Arithmetic Mean Filtering (AMF): This is one of the simplest of the mean filtering techniques. It expressed as AMF by the equation (4).[6]

$$
f(x, y)=\frac{1}{m n} \sum_{(a, t) \in s_{x y}} g(s, t)
$$

$\mathrm{S}_{\mathrm{xy}}$ represent the set of coordinates in a rectangular space that is image window of size $\mathrm{m} \mathrm{x} \mathrm{n}$ centred at given point $(\mathrm{x}, \mathrm{y})$. The AMF technique calculates the average value of the corrupted image $g(x, y)$ in the area defined by $S_{x y}$. The value of restored image $f$ at any point $(x, y)$ is Arithmetic Mean computed using the pixels values in the image that is in the region defined by $S_{x y}$.

ii). Median Filter: the median filter replaces the value of the pixel by the median of the gray levels in the neighbourhood of that pixel [1] i.e.

$$
Z_{(x, y)}=\text { median }_{(s, t) \in S_{x y}}\{g(x, y)\}
$$

The value of the pixel at (x,y) is included in the computation of the median. [7-9]

iii). Weiner filter: It is an adaptive low-pass filter which uses a pixel-wise adaptive Wiener method based on statistics estimated from a local neighbourhood of each pixel.[7] Adaptive filters are a class of filters which change their characteristics according to the values of the grey scales under the mask. Such a filter can be used to clean Gaussian noise by using local statistical properties of the values under the mask. One such filter is the minimum mean-square error filter; this is a non-linear spatial filter; and as with all spatial filters, is implemented by applying a function to the grey values under the mask. The output value can be calculated by following equation.[6]

$$
m_{f}+\frac{\sigma^{2}}{\sigma f^{2}+\sigma g^{2}}\left(g-m_{f}\right)
$$

Where; $\mathrm{g}$ is the current value of the pixel in the noise affected image, $\mathrm{mf}$ is the mean, $\sigma \mathrm{f} 2$ is the variance in the mask and $\sigma g 2$ is the variance of the noise over the entire image. The wiener2 
Multiple Noises Removal from Computed Tomography (CT) Images

function applies a Wiener filter which is a type of linear filter to an image adaptively, tailoring itself to local image variance. Where the variance is large, wiener2 performs little smoothing. Where the variance is small, wiener 2 performs more smoothing. This approach often produces better result than linear filtering. [8] The technique that is implemented in MATLAB is Weiner filter. It is an adaptive low-pass filter which uses a pixel-wise adaptive Wiener method based on statistics estimated from a local neighbourhood of each pixel.[7]

\section{Materials and Methods}

Brian and Chest Computed Tomography (CT) images of sizes $(225 \times 225)$ pixels are used in this study. These images are corrupted by multiple and different noises (speckle, Gaussian, salt and pepper). Filtering techniques (AMF, Median, and Weiner) are used to remove these noises. To evaluate filtering techniques quality metrics like Mean Squared Error (MSE), and Peak Signal to Noise Ratio (PSNR) were used. Algorithms were implemented in MATLAB with default setup parameters. The structure of methodology used in this study can be shown in Figure (3).

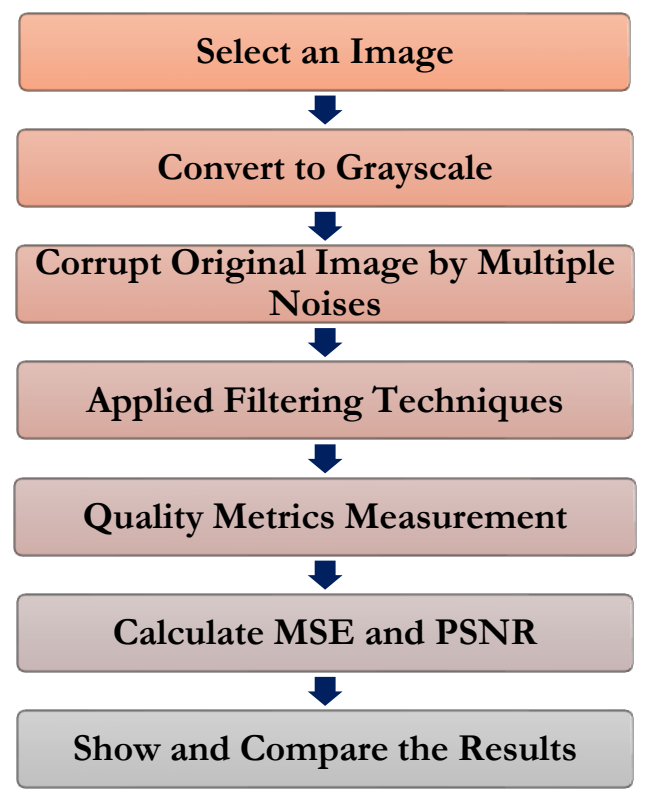

Figure 3: Methodology Structure

The (PSNR) is evaluated in decibels and is inversely proportional the Mean Squared Error. The PSNR can be calculated form equation (7) :[1]

$$
\operatorname{PSNR}(d B)=10 \log \left(\frac{(255)^{2}}{M S E}\right)
$$

Where (MSE) used to calculate by taking difference between two images pixel by pixel, and it is defined as :[3] 


$$
M S E=\frac{1}{M N} \sum_{i=1}^{M} \sum_{j=1}^{N}(x(i, j)-y(i, j))^{2}
$$

Where $x(i, j)$ represents the original (reference) image and $y(i, j)$ represents the distorted (modified) image and $i$ and $j$ are the pixel position of the $M \times N$ image. MSE is zero when $x(i$, j) $=y(i, j)$.

\section{Results and Discussion}

The visualization results of MATLAB simulation are shown in Figure (4).

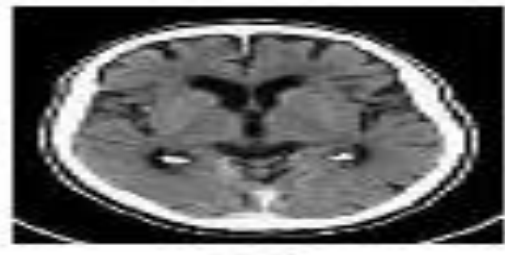

a

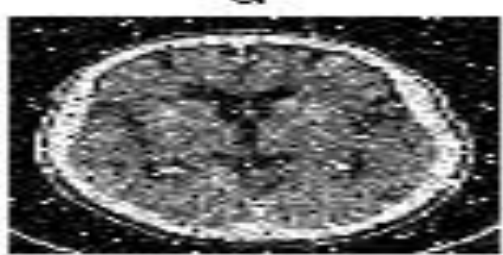

c

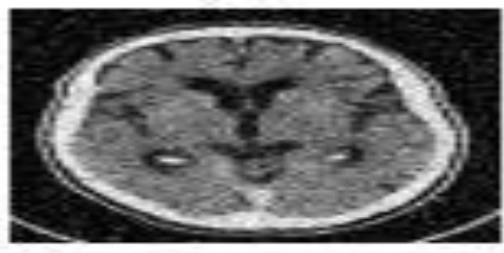

e

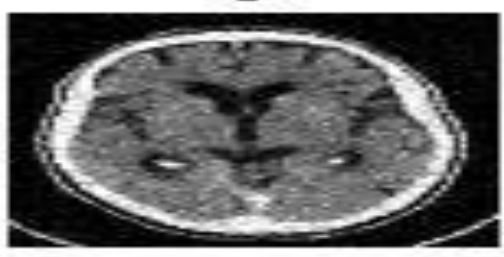

g

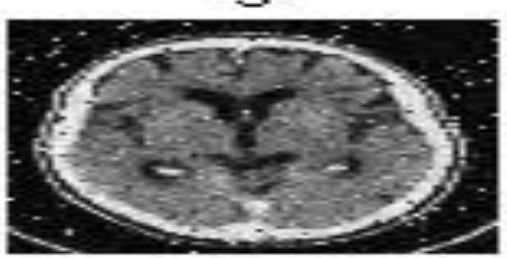

i

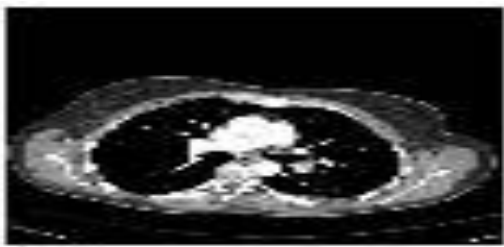

b

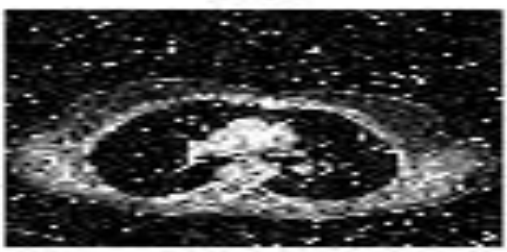

d

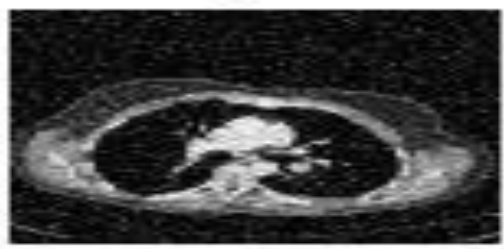

f

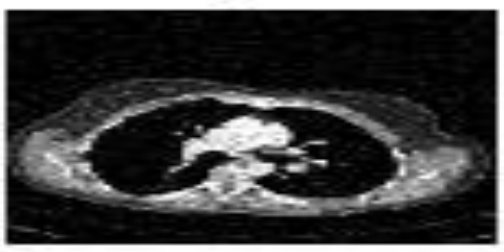

h

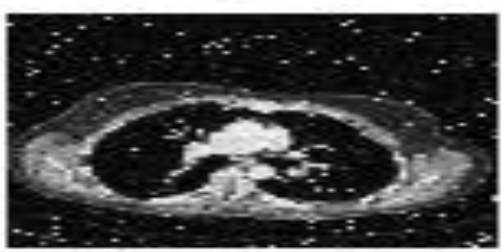

j

Figure 4: Filtering noisy (CT) images (a) and (b)original images(Brain \& Chest) (c) and (d) noisy images (e) and (f) Average filter applied (g) and (b) Median filtering applied (i) and (j) Weiner filtering applied 
Multiple Noises Removal from Computed Tomography (CT) Images

The values of (MSE \& PSNR) for applied filter techniques are tabulated in Table (1) to evaluate and compare the filtered (CT) images. The comparison has been made amongst (AMF, Median, and Weiner) filters. Table (1), Figure (4), and Figure (5). show the experimental results and illustrate a thorough comparison of all the filtering techniques for brain and chest (CT) image used in this study.

Table 1: MSE and PSNR values

\begin{tabular}{|c|c|c|c|c|}
\hline Image & \multicolumn{2}{|c|}{ Brain } & \multicolumn{2}{c|}{ Chest } \\
\hline Filter & MSE & PSNR & MSE & PSNR \\
\hline Average & 572.77 & 20.55 & 742.16 & 19.42 \\
\hline Median & 412.41 & 21.97 & 435.38 & 21.74 \\
\hline Weiner & 886.03 & 18.65 & $1.10 \times 10^{3}$ & 17.69 \\
\hline
\end{tabular}

Through finding the noises (speckle, Gaussian, salt and pepper) noises in both (CT) images by filtering techniques it is found that the median filter works better for the removing multiple noises from (CT) images. The highest values of (PSNR) are given by Median filter 21.97 and 21.74 for Brain and Chest (CT) images respectively. Therefore the output images is greater denoised. The median filter is more effective in removing different noises (speckle noise, Gaussian noise, salt and pepper). Whereas the Weiner filter can be used to clean Gaussian noise by using local statistical properties of the values under the mask, and it fails for salt and pepper noise. The median is much less sensitive than the mean to extreme values. Since the median value must actually be the value of one of the pixels in the neighbourhood, the median filter does not create new unrealistic pixel values when the filter straddles an edge. These advantages aid median filters in suppressing the uniform noise as well as other noises. Median filtering is therefore better able to remove this outlier without reducing the sharpness of the image.[7-9]

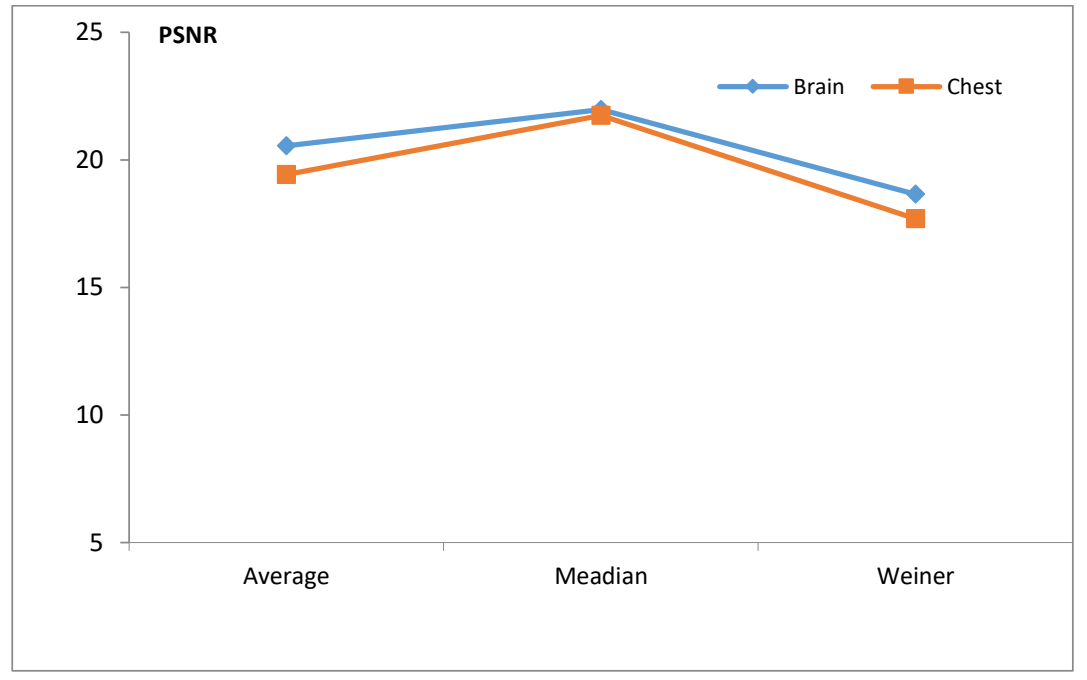

Figure 5: PSNR values 


\section{Conclusions}

During image acquisition and transmission, noise is seen in images. This is characterized by noise model. Image denoising is necessary action in image processing operation. Noise models also designed by probability density function using mean, variance and mainly gray levels in digital images. In this work, we have applied different filtering techniques (AMF, Median, and Weiner) to remove multiple noises (speckle noise, Gaussian noise, salt and pepper). The simulation results found that the median filter is more effective in removing different noises. Through this work we have observed that the choice of filters for denoising the medical images depends on the type of noise and type of filtering technique, which are used. These filters are very useful in many applications. These results are more useful for medical diagnostic.

\section{References}

[1] S.Senthilraja, P.Suresh, and M.Suganthi, "Noise Reduction in Computed Tomography Image Using WB - Filter", International Journal of Scientific \& Engineering Research, Volume 5, Issue 3, pp. 243-247, March-2014.

[2] Ajay Kumar Boyat, and Brijendra Kumar Joshi, "A Review Paper: Noise Models in Digital Image Processing", Signal \& Image Processing : An International Journal, SIPIJ, Vol. 6, No. 2, pp. 63-75, April- 2015.

[3] C.Sasi varnan, et al, "Image Quality Assessment Techniques on Spatial Domain", International Journal of Computer Science and Technology, Vol. 2, Issue 3, pp. 177-184, September 2011

[4] K. Somasundaram, and P. Kalavathi, "Medical Image Denoising using Non-Linear Spatial Mean Filters for Edge Detection", National Conference of Signal and Image Processing, (NCSIP), pp.149-153, 2012.

[5] SONALI R. MAHAKALE \& NILESHSINGH V. THAKUR "A Comparative Study of Image Filtering on Various Noisy Pixels", International Journal of Image Processing and Vision Sciences, Vol. 1, Issue 2, pp. 69-77, 2012.

[6] Gururaj P.Surampalli et al, "Adaptive Approach to Retrieve Image Affected by Impulse Noise", International Journal of Research in Engineering and Technology, Vol. 3, Special Issue 3, pp. 218-221, May- 2014.

[7] Ajay Kumar Nain, et al "A Comparative Study of Mixed Noise Removal Techniques", International Journal of Signal Processing, Vol. 7, No. 1, pp.405-414, 2014.

[8] Bhausaheb Shinde, Dnyandeo Mhaske, and A.R. Dani, "Study of Noise Detection and Noise Removal Techniques in Medical Images", I.J. Image, Graphics and Signal Processing, pp. 51-60, 2012.

[9] Sukhwinder Singh, and Neelam Rup Prakash, "Modified Adaptive Median Filter for Salt \& Pepper Noise" International Journal of Advanced Research in Computer and Communication Engineering, Vol. 3, Issue 1, pp. 5067-5071, January 2014. 